\title{
Clinical utility of SNP microarray in developmental delay: a rare genetic condition-potocki-lupski syndrome (PTLS) from India
}

\begin{abstract}
Many genetic syndromes with developmental delay/mental retardation have been described with characteristic phenotypes and behavioral features. In a resourcepoor country like India, it is not even feasible to conduct basic karyotype \& metabolic screening in children with developmental delay. Many cases are left undiagnosed due to lack of infrastructure or non-availability of advanced genomic technology at clinician's finger tip. Microarray-based whole genome analysis detects submicroscopic alterations in mentally retarded (MR) patients at higher resolution than routine chromosomal analysis. We report here, to the best of our knowledge for the first time in Indian population, a rare genetic case of Potocki-Lupski syndrome (PTLS) [MIM 610883] detected by SNP microarray which helped us further in genetic counseling \& prevention of recurrence risk in the family. A226 Kb duplication at cytoband 17 p12p11.2 with clinical significance and a copy neutral LOH (Loss of Heterozygosity) on chromosomes 11 and 15 representing about $0.25 \%$ of the genome was detected.
\end{abstract}

Keywords: genetic syndrome, developmental delay, dysmorphism, microarray, genetic counseling
Volume 4 Issue 2 - 2017

Usha P Dave, Priyankka Damani

MILS International India, India

Correspondence: Usha P. Dave, MILS International India, 504 E, BWing, Summit Samarth Arcade, Off Aarey Road, Goregaon (W), Mumbai-400062, Maharashtra, India, Tel +91 9820693161, Email dr.daveusha@gmail.com; milsindia.int@gmail.com

Received: September 24, 2016 | Published: March 21, 2017

\section{Introduction}

Despite a strong clinical diagnosis of genetic origin in a patient with developmental delay/mental retardation, the basic genetic investigations such as chromosomal karyotype \& IEM metabolic profiling can turn out to be normal. This leads to a clinician's dilemma \& it is difficult to face the parents \& family of children with developmental delay as no precise causative factor is detected. However, with the advancement of genomic technology, further genetic investigations are now possible, e.g. cytogenetic microarray (CMA) can detect the $<5-10 \mathrm{Mb}$ chromosomal aberrations, which are otherwise missed during routine karyotyping. Several genetic syndromes with mental retardation (MR) reveal submicroscopic chromosomal abnormalities ascertaining the precise diagnosis, so very important in genetic counseling.

Potocki-Lupski syndrome (PTLS [MIM 610883]) is a recently recognized, $17 \mathrm{p} 11.2$ duplication-associated condition with clinical features including infantile hypotonia, failure to thrive, mental retardation, autism, behavioral abnormalities, sleep apnea, and structural cardiovascular anomalies. ${ }^{1,2}$ The condition is also associated with a wide range of congenital anomalies such as ophthalmic, orthopedic, oral-pharyngeal, and renal abnormalities, microcephaly, distinct facial features, including pronounced nose, ears, and forehead and geometrical (triangular or square) faces, and a number of cognitive and behavioral indicators of developmental delay. ${ }^{3}$ We report here, to the best of our knowledge for the first time in Indian population, a three years old female with developmental delay, mild autistic features, dimorphism and multiple congenital anomalies \& who is diagnosed on microarray analysis as Potocki-Lupski syndrome.

\section{Case report}

A three year old female was presented with developmental delay and Dysmorphism (Figure 1). She was the second child born of non-consanguineous endogamous marriage. She was delivered after 9months and 9days of gestation by caesarean section for indication of non progression of labor. Her birth weight was average and showed no signs of perinatal asphyxia. The Informed parent consent for a research study according to the ethical guidelines of Helsinki Declaration-2013 and ICMR (Indian Counsel of Medical Research), 2006 for biomedical research was obtained from the parents who cooperated for genomic studies.

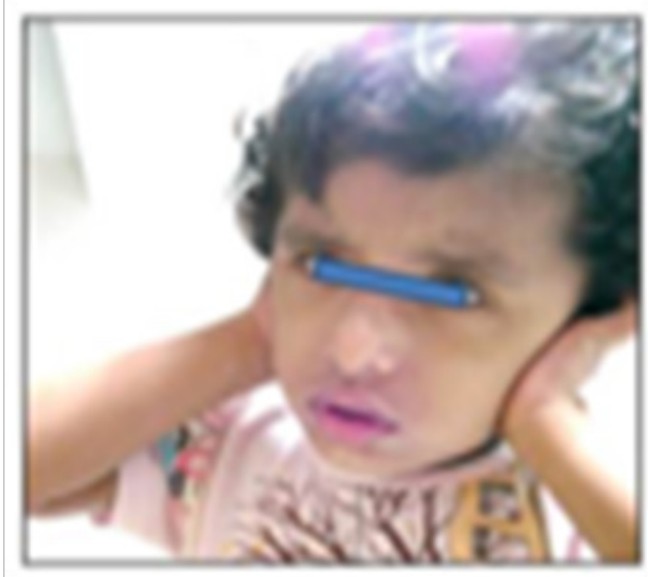

Figure I Facial features of the patient showing dysmorphis. 
Clinical examination revealed craniofacial dysmorphism and multiple congenital anomalies. She had squint since birth and vision assessment showed amblyopia and anisometropia and morning glory disc anomaly for which she was prescribed with spectacles for constant use. She showed no fixation of eyes and had constant horizontal sensory nystagmus. Her electroretinogram showed no evidence of retinal disease in both eyes.

Her milestones were delayed and had started walking with support six months before presentation. She showed an inconsistent response to name calling. She was assessed using the capute scales where representational play and object permanence was present. Her linguistic skills were at eight to ten months level. Repetitive behaviors and tactile seeking behaviors were noted. Physical examination revealed hypotonia, soft palate fissure, microphthalmia, microcornea, frontal bossing, anteverted nares, small ears and syndactyly of left thumb.

Magnetic Resonance Imaging (MRI) of the brain was done on a $3 \mathrm{~T}$ system in axial, coronal and sagittal planes with $5 \mathrm{~mm}$ slice thickness. The cerebral cortex was unremarkable. The Corpus callous was found to be intact, cerebellar vermis was normal and there was no evidence of pachygyria or polymicrogyria. No significant findings were identified on brain MRI studies.

\section{Cytogenetic analysis}

The chromosomal analysis using peripheral leucocytes revealed a normal female $(46, \mathrm{XX})$ karyotype with no structural or numerical aberrations at the light microscopy level.

\section{High-risk metabolic screening}

Dried blood spot (DBS) specimen and urine was collected for high-risk screening for congenital metabolic abnormalities using Tandem Mass Spectrometry (TMS) and Gas Chromatography/Mass Spectrometry (GC/MS) respectively. TMS analysis for 46 common conditions was performed $\&$ the metabolic screen revealed a normal blood amino acid (aminoacidopathies) and acylcarnitine profiles (organic acidemias and fatty acid oxidation disorders). Matsumoto's method was used for urinary analysis of $100+$ congenital metabolic disorders involving organic acids, amino acids, sugars, sugar acids, sugar alcohols, nucleic acids and nucleic acid bases. ${ }^{4}$ Urinary metabolic profiling showed normal levels of amino acids and organic acids \& no abnormal markers were detected. Thus, common genetic metabolic conditions were ruled out in this case.

\section{SNP Microarray analysis}

SNP microarray analysis was performed using Illumina HumanCytoSNP-12 array chip (Illumina, Inc, USA) with approx. $3,00,000$ probes across the whole genome that are designed to target SNP markers for regions prone to chromosomal aberrations. Imbalances as small as $30 \mathrm{~kb}$ with an average marker spacing of $6.2 \mathrm{~kb}$ were screened for 250 cytogenetic abnormality regions. De novo imbalances related to common micro deletion/micro duplication syndromes, subtelomeric deletions or duplications, and at several loci throughout the genome were studied. The chip also detects Copy Neutral Loss of Heterozygosity $(\mathrm{CN}-\mathrm{LOH})$. Karyostudio software (Illumina, Inc., USA) was used for visualizing and analyzing arraybased cytogenetic data. The test cannot detect single gene disorders (Mutational sites) \& balanced translocation, inversion and gene conversion. Copy number changes that do not contain genes and also smaller than $0.2 \mathrm{Mb}$ (unless associated with a gene of known clinical significance) were omitted. Only copy neutral $\mathrm{LOH}>5 \mathrm{Mb}$ with clinical significance were interpreted.

The results showed a female karyotype with a copy number change, duplication at cytoband $17 \mathrm{p} 12 \mathrm{p} 11.2$ with clinical significance and also revealed a copy neutral LOHs on chromosomes 11 and 15 . Duplication of $226 \mathrm{~Kb}$ at cytoband $17 \mathrm{p} 12 \mathrm{p} 11.2$ was detected (Figure 2). Also, long continuous stretch of copy neutral LOH (Loss of Heterozygosity) on chromosomes 11 and 15 representing about $0.25 \%$ of the genome was observed (Figure 3). Other three small aberrations in the form of deletions were seen in chromosomes 5, 7 and 8 .

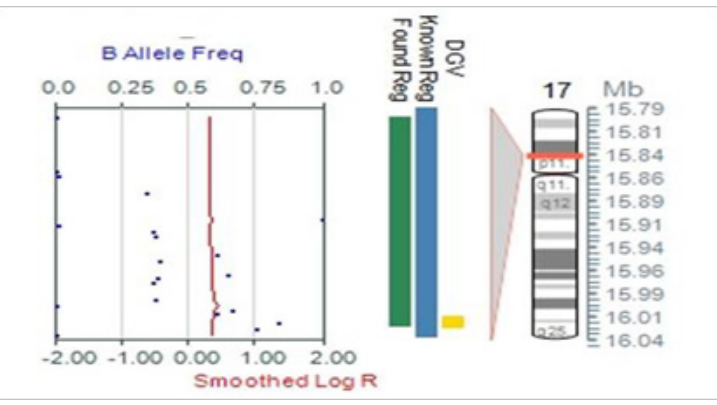

Figure 2 A copy number change, duplication on chromosome 17 at cytoband $|7 p| 2 p \mid 1.2$

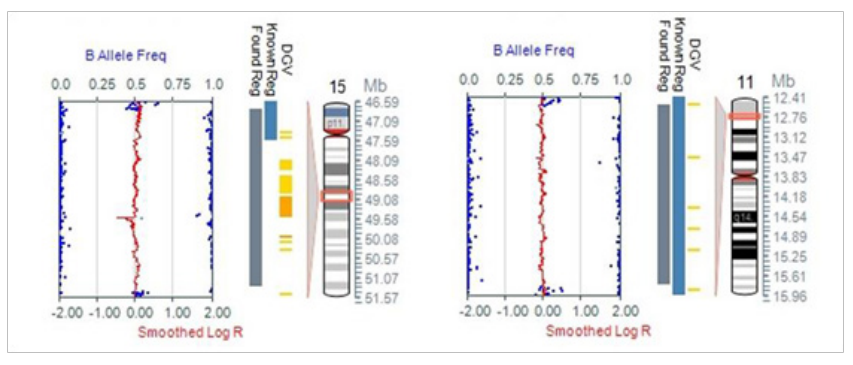

Figure 3 A copy neutral LOH (loss of heterozygosity) on chromosomes II and $\mathrm{I} 5$.

\section{Discussion}

The Potocki-Lupski syndrome, associated with dup (17) (p11.2p11.2), is a recently recognized syndrome of multiple congenital anomalies and mental retardation. It is the first predicted reciprocal microduplication syndrome described as the chromosomal duplication represents the predicted non-allelic homologous recombination (NAHR) reciprocal of the Smith-Magenis syndrome (SMS) microdeletion (del(17)(p11.2p11.2))..$^{5}$ Molecular studies suggest potential mechanistic differences between nonrecurrent duplications and nonrecurrent genomic deletions. PTLS is clinically recognizable and is associated with hypotonia and failure to thrive in infancy and early childhood, developmental delay, mental retardation, severe communication disorder, autistic spectrum disorder, sleep disordered breathing, EEG abnormalities, cardiovascular anomalies, and hypermetropia. However, no major dosage-sensitive gene of consequence has been identified in PTLS.

The majority of PTLS patients identified to date harbor a causative common $3.7 \mathrm{Mb}$ duplication copy number variant $(\mathrm{CNV})$ generated by NAHR between the distal and proximal SMSREPs; however, uncommon recurrent duplications, non-recurrent 
PTLS rearrangements, and duplications associated with marker chromosomes, have also been described. ${ }^{1,6-8}$ Duplications, as small as $125 \mathrm{~kb}$, containing only the RAI1 gene have been identified. Nonallelic homologous recombination (NAHR) between region-specific lowcopy repeats (LCRs) (also known as "segmental duplications") is a major cause of DNA rearrangements associated with many genomic disorders..$^{9,10}$ The proximal short arm of $17 p$ is particularly rich in LCRs and is thus susceptible to duplications that may lead to genetic disorders. ${ }^{1}$

Total six aberrations were seen in the present case. One $0.2 \mathrm{Mb}$ duplication in the proximal short arm of $17 \mathrm{p}$ was noted. Thus, the reported duplicated region is larger than the above mentioned size. However, the spectra of clinical features depend on the size of the duplication. The copy neutral LOH seen in chromosome 11 and 15 may be associated with a risk of uniparental disomy and recessive diseases. In general, the copy neutral LOH regions in the genome represent identity by descent. The other three aberrations in the form of deletions seen in chromosomes 5,7 and 8 were with no known clinically significant genetic condition.

Before the development of microarrays the cause of mental retardation in many cases was considered to be non-specific or idiopathic due to lack of sufficient technology to diagnose genetic disorders. However, microarrays have revolutionized diagnostics by providing means to correlate genetic imbalances with mental retardation and other neurological conditions. Microarrays can simultaneously test for hundreds of genetic disorders caused by rare genetic changes. Thus, it is particularly helpful in diagnosis of rare genetic conditions where other genetic tests like PCR and RT-PCR are not useful for testing many disorders simultaneously.

A major cause of the genetic variation in individuals is because of genomic rearrangements like copy number variations including deletions and duplications. So far 50cases of Potocki-Lupski syndrome (PTLS) have been described in the medical literature but to the best of our knowledge, none from India. Microarrays are gaining importance in diagnosis of rare genetic disorders when conventional karyotype \& other metabolic genetic tests are not useful, \& where specific medical follow-up of the patient is essential. In the present case, SNP microarray analysis could offer the precise diagnosis, and further genetic counseling was feasible to prevent the recurrence risk in the family.

\section{Acknowledgements}

The authors are grateful to parents to cooperate $\&$ sincerely thank PG student-Umakant Nadkar \& all the R \& D staff for their help to conduct this study.

\section{Conflict of interest}

There is no conflict of interest between authors \& no funding was received to conduct this study.

\section{References}

1. Potocki L, Bi W, Treadwell-Deering D, et al. Characterization of PotockiLupski syndrome $(\operatorname{dup}(17)(\mathrm{p} 11.2 \mathrm{p} 11.2)$ and delineation of a dosagesensitive critical interval that can convey an autism phenotype. Am J Hum Genet. 2007;80(4):633-649.

2. Treadwell-Deering DE, Powell MP, Potocki L. Cognitive and behavioral characterization of the Potocki Lupski syndrome (duplication 17p11.2). J Dev Behav Pediatr. 2010;31(2):137-43.

3. Gulhan Ercan-Sencicek A, Davis Wright NR, Frost SJ, et al. Searching for Potocki-Lupski syndrome phenotype: A patient with language impairment and no autism. Brain Dev. 2012;34(8):700-703.

4. Matsumoto I, Kuhara T. A new chemical diagnostic method for inborn errors of metabolism by mass spectrometry-rapid, practical and simultaneous urinary metabolites analysis. Mass Spectrometry Reviews. 1996;15(1):43-57.

5. Sanchez-Valle A, Pierpont ME, Potocki L. The severe end of the spectrum: hypoplastic left heart in Potocki-Lupski syndrome. Am J Med Genet A. 2011;155A(2):363-366.

6. Yatsenko SA, Treadwell-Deering D, Krull K, et al. Trisomy 17p10-p12 due to mosaic supernumerary marker chromosome: Delineation of molecular breakpoints and clinical phenotype, and comparison to other proximal $17 \mathrm{p}$ segmental duplications. Am J Med Genet. 2005;138A(2):175-180.

7. Zhang F, Khajavi M, Connolly AM, et al. The DNA replication FoSTeS/ MMBIR mechanism can generate human genomic, genic, and exonic complex rearrangements. Nat Genet. 2009;41(7):849-853.

8. Zhang F, Potocki L, Sampson JB, et al. Identification of uncommon recurrent Potocki-Lupski syndrome-associated duplications and the distribution of rearrangement types and mechanisms in PTLS. Am J Hum Genet. 2010;86(3):462-470.

9. Lupski JR. Genomic disorders: structural features of the genome can lead to DNA rearrangements and human disease traits. Trends Genet. 1998;14(10):417-422

10. Stankiewicz P, Lupski JR. Genome architecture, rearrangements and genomic disorders. Trends Genet. 2002;18(2):74-82. 